\title{
REMARKS ON AN ARITHMETIC DERIVATIVE
}

\section{E. J. Barbeau}

(received March 21, 1961) variable $\mathrm{n} \geq 0$ such that

(1) $\mathrm{D}(1)=\mathrm{D}(0)=0$

(2) $D(p)=1$ for every prime $p$

(3) $D\left(n_{1} n_{2}\right)=n_{1} D\left(n_{2}\right)+n_{2} D\left(n_{1}\right)$ for every pair of non-negative integers $n_{1}, n_{2}$.

The property (3) is analagous to the product rule for derivatives, and its extension to $\mathrm{k}$ terms

(4) $D(n)=n \sum_{i=1}^{k} n_{i}^{-1} D\left(n_{i}\right)$ for $n=n_{1} n_{2} \cdots n_{k}$

is immediate. - The above properties are consistent and determine $D(n)$ uniquely for all non-negative integers $n$. In fact, if $\mathrm{n}=\mathrm{p}_{1}^{\alpha_{1}} \mathrm{p}_{2}^{\alpha_{2}} \ldots \mathrm{p}_{\mathbf{r}}^{\alpha_{\mathrm{r}}}$, we have, on using (4),

$$
D(n)=n \sum_{i=1}^{r} \alpha_{i} p_{i}^{-1}
$$

so that, once the prime factor decomposition of $\mathrm{n}$ is known, the first derivative $D(n)$ is given explicitly. However, the "higher" derivatives, defined successively by

$D^{0}(n)=n, D^{1}(n)=D(n), D^{2}(n)=D[D(n)], \ldots, D^{k}(n)=D\left[D^{k-1}(n)\right]$

\footnotetext{
${ }^{1}$ I have not been able to trace explicit references to previous work on $D(n)$. However, it appeared in a question on the Putnam Prize competition (1950); see American Mathematical Monthly 57 (1950), p. 469. I am indebted to Dr. J.H.H. Chalk for suggesting a note on this topic and for assistance during its preparation.
}

Canad. Math. Bull. vol. 4, no. 2, May 1961. 
present an unsolved problem. For fixed $n$, the function $D^{k}(n)$ of $k$ exhibits irregular behaviour as $k$ increases. For example, using (3) with $n=p^{p} n_{1}$, where $p$ is a prime, we obtain

$$
D(n)=p^{p}\left[n_{1}+D\left(n_{1}\right)\right] \geq n
$$

equality holding if and only if $n_{1}=1$. Hence, for integers $n$ possessing a proper divisor of the form $\mathrm{p}^{\mathrm{p}}$, $\operatorname{Iim} D^{\mathrm{k}}(\mathrm{n})=\infty$, and if $n=p p, D^{k}(n)=n$ for all $k$. On the other hand, $D^{k}(p)=0$ for all $k>1$ and all primes $p$. Numerical considerations suggest the following.

CONJECTURE. For each $n>1$, the re exists a constant $k_{0}=k_{0}(n) \geq 1$ such that, for all $k \geq k_{0}$,

either

$$
\text { 1) } D^{k}(n)=0
$$

OI

2) $D^{k}(n) \neq 0$,

and there exists a prime $p$ such that $D^{k}(n) \equiv 0(\bmod p)$.

2. Some remarks about $D(n)$. Although the function $D(n)$ behaves erratically, it is easy to obtain exact upper and lower bounds, depending on $n$, for its values. We suppose that $n=q_{1} q_{2} \ldots q_{\nu}$ has prime factors $q_{i}$ which are not necessarily distinct.

(a) $D(n) \leq \frac{n \log n}{2 \log 2}$ for all $n$, equality occurring if and only if $n$ is a power of 2 . In fact, $n$ satisfies $2^{k} \leq n<2^{k+1}$ for some $k$. Clearly, $v \leq k$ and

$$
D_{(n)}(n) \sum_{i=1}^{v} \frac{1}{q_{i}} \leq n \sum_{i=1}^{v} \frac{1}{2} \leq \frac{n k}{2} \leq \frac{n \log n}{2 \log 2} .
$$

If $n=2^{k}, D(n)=k 2^{k-1}=\frac{2^{k} \log 2^{k}}{2 \log 2}$. If $n \neq 2^{k}$, then some $q_{i} \neq 2$ and strict inequality holds in the above.

(b) $D(n) \geq v n^{1-\frac{1}{v}}$, equality holding if, and only if, all the factors $q_{i}$ are equal. For, by (5) and the inequality of the arithmetic and geometric means, 


$$
D(n)=n \Sigma_{i=1}^{v} \frac{1}{q_{i}} \geq n v \frac{1}{\left(q_{1} q_{2} \cdots q_{\nu}\right)^{\frac{1}{v}}}=v n^{1-\frac{1}{v}}
$$

Hence, if $n$ is not a prime or unity, $D(n) \geq 2 \sqrt{n}$, with equality if and only if $n=p^{2}$ where $p$ is a prime.

In addition, we can relate the value of $D(n)$ to $n$ in the following ways.

(c) Let $n=p_{1}^{\alpha_{1}} \ldots p_{r}^{\alpha}$, where $p_{1}, \ldots, p_{r}$ are distinct primes. Then $D(n) \equiv 0(\bmod n)$ if, and only if, $\alpha_{1} \equiv 0\left(\bmod \mathrm{p}_{1}\right), \ldots, \alpha_{\mathrm{r}} \equiv 0\left(\bmod \mathrm{p}_{\mathrm{r}}\right)$. In particular, $\mathrm{D}(\mathrm{n})=\mathrm{n}$ if and only if, $n=p^{p}$. The sufficiency of the conditions is obvious.

Their necessity is seen by noting that, if $n=p^{\alpha} n^{\prime}$, where $\left(p, n^{\prime}\right)=1$, then $\mathrm{D}(\mathrm{n})=\mathrm{n}^{\prime} \alpha \mathrm{p}^{\alpha-1}+\mathrm{p}^{\alpha} \mathrm{D}\left(\mathrm{n}^{\prime}\right) \equiv 0(\bmod \mathrm{n})$ implies $n^{\prime} \alpha p^{\alpha-1}=0\left(\bmod p^{\alpha}\right)$ and, hence, $\alpha \equiv 0(\bmod p)$, since $\left(n^{\prime}, p\right)=1$.

(d) If $D(n) \geq n$, then $D(k n)=k D(n)+n D(k)>k n$ for aII $k>1$.

3. The average order of $\mathrm{D}(\mathrm{n})$. Let

$$
S(n)=\sum_{r=1}^{n} D(r), \quad T(n)=\sum_{r=1}^{n} K(r)
$$

where $K(n)=n^{-1} D(n)$. Since $K(n)$ is totally additive, i.e. $\mathrm{K}\left(\mathrm{n}_{1} \mathrm{n}_{2}\right)=\mathrm{K}\left(\mathrm{n}_{1}\right)+\mathrm{K}\left(\mathrm{n}_{2}\right)$ for all integer pairs $\mathrm{n}_{1}, \mathrm{n}_{2}$, it is easier to estimate $T(n)$ first, and then use partial summation to deduce the average order of $D(n)$. Let

$$
j(n, p)=\Sigma_{t=1}^{\infty}\left[\frac{n}{p^{t}}\right], \alpha(n)=\left[\frac{\log n}{\log 2}\right] ;
$$

then $j(n, p)$ denotes $[1 ; p .342]$ the exponent of the highest power of $p$ dividing $n$ ! and $\alpha(n)$ denotes the exponent of the highest power of $2 \leq n$. Observe that

$$
\begin{aligned}
T(n)=K(n !) & =\Sigma_{p \leq n} \frac{1}{p} j(n, p) \\
& =\Sigma_{p \leq n} \frac{1}{p}\left(\Sigma_{t=1}^{\infty}\left[\frac{n}{p^{t}}\right]\right)
\end{aligned}
$$




$$
\begin{aligned}
& =\Sigma_{p \leq n} \frac{1}{p}\left(\Sigma_{t=1}^{\alpha(n)}\left[\frac{n}{p^{t}}\right]\right) \\
& =\Sigma_{p} \leq n \frac{1}{p}\left\{\Sigma_{t=1}^{\alpha(n)} \frac{n}{p^{t}}+O(\log n)\right\} \\
& =\Sigma_{p \leq n}\left\{\Sigma_{t=2}^{\infty} \frac{n}{p^{t}}-\Sigma_{\alpha(n)+2}^{\infty} \frac{n}{p^{t}}\right\}+O\left\{(\log n) \Sigma p \leq n \frac{1}{p}\right\} \\
& =n \Sigma_{p \leq n} \frac{1}{p(p-1)}-\Sigma_{p \leq n} \frac{1}{p^{\alpha(n)+1}(p-1)}+O\left\{(\log n) \sum_{p} \leq n \frac{1}{p}\right\} \\
& =\mathrm{n} \sum_{\mathrm{p}=2}^{\infty} \frac{1}{\mathrm{p}(\mathrm{p}-1)}-\Sigma_{\mathrm{p}>\mathrm{n}} \frac{\mathrm{n}}{\mathrm{p}(\mathrm{p}-1)}-\Sigma_{\mathrm{p}} \leq \mathrm{n} \frac{\mathrm{n}}{\mathrm{p}^{\alpha(\mathrm{n})+1}(\mathrm{p}-1)} \\
& +O\left\{(\log n) \Sigma_{p \leq n} \frac{1}{p}\right\} \\
& =T_{0} n+O\{(\log n)(\log \log n)\}
\end{aligned}
$$

where $T_{0}=\sum_{p=2}^{\infty} \frac{1}{p(p-1)}=0.749 \ldots$

since

$$
\begin{gathered}
\sum_{\mathrm{p}>\mathrm{n}} \frac{\mathrm{n}}{\mathrm{p}(\mathrm{p}-1)}<\mathrm{n} \Sigma_{\mathrm{k}}>\mathrm{n} \frac{1}{\mathrm{k}(\mathrm{k}-1)} \leq 1, \\
\mathrm{p}^{\alpha+1}>\mathrm{p}^{\frac{\log \mathrm{I} \log 2}{\log n}} \geq 2^{\frac{\log 2}{\log } \geq \mathrm{n},} \\
\Sigma_{\mathrm{p} \leq \mathrm{n}}\left\{\frac{1}{\mathrm{p}-1}-\frac{1}{\mathrm{p}}\right\} \leq 1, \\
\Sigma_{\mathrm{p} \leq \mathrm{n}} \frac{1}{\mathrm{p}}=\mathrm{O}(\log \log \mathrm{n}) .
\end{gathered}
$$

For $S(n)$, we have

$$
\begin{aligned}
S(n) & =\sum_{r=1}^{n} r K(r)=T(n)+\Sigma_{r=1}^{n-1}\{T(n)-T(r)\} \\
& =n T(n)-\Sigma_{r=1}^{n-1} T(r) \\
& =n\left\{T_{0} n+O\left(n^{\delta}\right)\right\}-T_{0} \Sigma_{r=1}^{n-1} r+O\left(n^{1+\delta}\right) \\
& =T_{0} n^{2}-T_{0} \frac{n(n-1)}{2}+O\left(n^{1+\delta}\right)
\end{aligned}
$$




$$
=\frac{1}{2} T_{0} n^{2}+O\left(n^{1+\delta}\right)
$$

where $\frac{1}{2} T_{0}=0.374 \ldots$, for each fixed $\delta>0$.

4. The congruence $D(n) \equiv 0(\bmod 4)$. A key problem is to find a characterization of those numbers for which $\lim _{k \rightarrow \infty} D^{k}(n)=\infty$. This limit is known for numbers $n$ of the form $p, p p, k p p$ where $p$ is any prime. Further investigation is hampered by the absence of explicit formulae for the higher derivatives. If there were some way of dealing with $D(m+n)$ for any integers $m$ and $n$, then $D^{2}(n)$ could be determined from $D(n)=\Sigma \sum_{i=1}^{k} F_{i}$, where $n=\prod_{i=1}^{k} f_{i}, F_{i}=n / f_{i}, f_{i}$ prime. However, it is known only that, if $D(m+n)=D(m)+D(n)$, then $D(k m+k n)=D(k m)+D(k n)$ for every integer $k$; in particular, $D(h)+D(2 h)=D(3 h)$.

Another approach to the problem is to find a characterization of those numbers, excluding $p, p p, k p p$ for which $p^{p} / D^{k}(n)$ for some positive integer $k$ and some prime $p$. According to our conjecture, this would be sufficient to characterize those numbers for which $D^{k}(n) \rightarrow \infty$ as $k \rightarrow \infty$, provided $D^{k}(n) \neq 0$ for all $k$. We deal with the special case $p=2, k=1$.

Let $n=2^{\alpha} p_{1} p_{2} \ldots p_{r} q_{1} q_{2} \ldots q_{s}$ where $p_{i} \equiv 1(\bmod 4)$, $q_{j} \equiv-1(\bmod 4)$ are primes, not necessarily distinct. We have the following results:

(i) if $\alpha=0$, then $D(n) \equiv(-1)^{s}(r-s)\left(\bmod 2^{2}\right)$

(ii) if $\alpha=1$, then $\mathrm{D}(\mathrm{n}) \equiv(-1)^{\mathrm{s}}[1+2(\mathrm{r}-\mathrm{s})] \equiv(-1)^{\mathrm{r}-1}\left(\bmod 2^{2}\right)$ (iii) if $\alpha>1$, then $D(n) \equiv 0\left(\bmod 2^{2}\right)$.

In order to prove (i), let $P=p_{1} p_{2} \cdots p_{r} \equiv(+1)(\bmod 4)$

$$
\begin{aligned}
& Q=q_{1} q_{2} \cdots q_{s} \equiv(-1)^{s}(\bmod 4) \\
& P_{i}=\frac{P}{P_{i}} \equiv 1(\bmod 4) \\
& Q_{i}=\frac{Q}{q_{i}} \equiv(-1)^{s-1}(\bmod 4) .
\end{aligned}
$$

2 The approximation $0.374 \ldots n^{2}$ for $S(n)$ is good, even for small values of $n$. For example, $S(10)=38 \doteqdot(0.374 \ldots)(100)$. 
Then

$$
\begin{aligned}
D(n) & =D(P Q)=\sum_{i=1}^{r} P_{i} Q+\sum_{i=1}^{s} P Q_{i} \equiv r(-1)^{s}+s(-1)^{s-1} \\
& \equiv(-1)^{s}(r-s)(\bmod 4) .
\end{aligned}
$$

In case (ii),

$$
\begin{aligned}
D(2 P Q) & =P Q D(2)+2 D(P Q) \\
& \equiv(-1)^{s}+2(-1)^{s}(r-s) \\
& \equiv(-1)^{s}[1+2(r-s)](\bmod 4)
\end{aligned}
$$

Result (iii) follows from the fact that $4 / \mathrm{n}$. We conclude that $D(n) \equiv 0(\bmod 4)$ if and only if

(a) $\alpha=0, r \equiv s(\bmod 4)$

(b) $\quad \alpha>1$.

The numbers in (a) have a density of $\frac{1}{8}$ in the integers; those in (b) have a density of $\frac{1}{4}$. Hence, those integers $n$ satisfying $\lim _{k \rightarrow \infty} D^{k}(n)=\infty$ (which include the numbers of $(a)$ and $(b)$ ) have a density exceeding $\frac{3}{8}$. What this density is remains an open que stion.

\section{REFERENCE}

1. G. H. Hardy and E. M. Wright, Introduction to the Theory of Numbers, 4th edition, (Oxford, 1960).

University of Toronto 